\title{
Synthesis and biological evaluation of novel benzothiophene derivatives
}

\author{
MUHEB A S ALGSO ${ }^{\mathrm{a}}$, ARIF KIVRAK ${ }^{\mathrm{a}, *}$, METIN KONUS $^{\mathrm{b}}$, CAN YILMAZ $^{\mathrm{b}}$ and \\ ASLIHAN KURT-KIZILDOĞAN ${ }^{\mathrm{c}}$

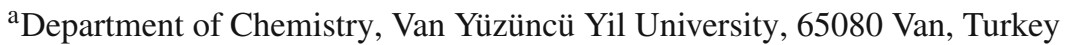 \\ ${ }^{\mathrm{b}}$ Department of Molecular Biology and Genetics, Van Yüzüncü Yil University, 65080 Van, Turkey \\ ${ }^{\mathrm{c}}$ Department of Agricultural Biotechnology, Faculty of Agriculture, Ondokuz May1s University, Samsun 55139, \\ Turkey \\ E-mail: akivrak@yyu.edu.tr
}

MS received 30 March 2018; revised 23 June 2018; accepted 7 July 2018; published online 11 August 2018

\begin{abstract}
Benzothiophene derivatives were synthesized regioselectively using coupling reactions and electrophilic cyclization reactions. Antimicrobial properties of isolated compounds were tested against indicator microorganisms such as C. albicans ATCC 10231, B. subtilis ATCC 6633, E. coli ATCC 25922 and S. aureus ATCC 25923. 3-(4-aminobenzoethynyl)-2-(thiophen-2-yl) benzo[b]thiophene (12E), 3-ethynyl-2-(thiophen2-yl) benzo[b]thiophene (12L) and 3-(2-aminobenzoethynyl)-2-(thiophen-2-yl) benzo[b]thiophene (12J) displayed high antibacterial activity against $S$. aureus. Further, 3-iodo-2-(thiophen-2-yl) benzo[b]thiophene (10) and 3-(trimethylsilylethynyl)-2-(thiophen-2-yl) benzo[b] thiophene $(\mathbf{1 2 K})$ were found to have potentials to be used as antifungal agents against current fungal diseases. Novel 3-(1H-indole-2-yl)-2-(thiophen-2-yl) benzo[b] thiophene (16) and 3-(4-aminobenzoethynyl)-2-(thiophen-2-yl) benzo[b] thiophene (12E) also showed quite high antioxidant capacities with TEAC values of 2.5 and 1.1, respectively; which surpassed the antioxidant capacity of an universally accepted reference of trolox.
\end{abstract}

Keywords. Benzothiophenes; heteroaromatic compounds; biological properties; antioxidant capacity; antimicrobial agents.

\section{Introduction}

Heteroaromatic compounds have very important roles in the discovery and development of new drug candidates due to their biological and pharmacological properties. ${ }^{1,2}$ They have been used as anti-parasitic, ${ }^{3}$ anti-bacterial, ${ }^{4}$ anti-cancer, ${ }^{5}$ anti-fungal, ${ }^{6}$ anti-inflammatory ${ }^{7}$ and antioxidant ${ }^{8}$ drugs for years. In addition, they are known as strong inhibitors of lipid peroxidation, ${ }^{9}$ potassium channel openers, ${ }^{10}$ topoisomerase inhibitors, ${ }^{11}$ and L1210 cell selectors. ${ }^{12}$

Benzothiophenes and thiophenes are well-known candidates of heteroaromatic compounds and used as pharmaceuticals like Raloxifene 1, ${ }^{13}$ Zileuton 2, ${ }^{14}$ and Sertaconazole $\mathbf{3}$ (Scheme 1). ${ }^{15}$ Raloxifene, whose commercial brand name is Evista, is used for the treatment of breast cancer. Moreover, Raloxifene has

\footnotetext{
*For correspondence
}

less side-effects than another popular anti-cancer drug, Tamoxifen, with similar biological properties. ${ }^{16}$ Recently, a number of 3-(4-pyridinyl) amino benzothiophenes, which were selective serotonin re-uptake inhibitors, have been tested for their contribution on the treatment of central nervous system disorders such as Alzheimer's disease. ${ }^{17}$ Therefore, synthetic and medicinal chemists have tried to find novel organic compounds containing sulfur atoms for decades.

Over the past years, benzothiophenes have been generally obtained from intramolecular cyclization and Claisen rearrangement reactions. For example, benzothiophenes were regioselectively synthesized via intramolecular cyclization reaction of $o$-alkinylthioanisoles. ${ }^{18}$ Recently, Mohanakrishnan et al., published a new method for the synthesis of benzothiophene and dibenzothiophene starting from thiophenes and 2,5dimethoxy-THF using Lewis Acid catalyst. ${ }^{19}$ 

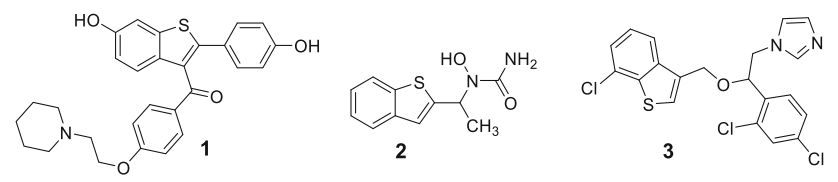

Scheme 1. Structures of Raloxifene 1, Zileton 2 and Sertaconazole 3.

Antimicrobial resistance to discovered antibiotics and other drugs used in the treatment of bacterial, fungal and viral infections is a serious problem threatening all the world population. Antimicrobial agents with unique chemical structures and strong antimicrobial potentials are urgently needed. However, the discovery rate of biologically active compounds has decreased. In this respect, chemically designed and synthesized sulfur containing benzothiophene compounds are among the promising members for the treatment of infections. ${ }^{20}$ Further, those benzothiophene derivatives have been tested and used as antioxidant agents for years. Antioxidants have essential roles in overall metabolism because of their potential in repairing damages generated by reactive oxygen species (ROS) and simple deactivation of those active compounds. Therefore, they are participants in hindering and reprieving various diseases such as cancer, ${ }^{21}$ diabetes, ${ }^{22}$ and cardiovascular problems. ${ }^{23,24}$ Besides natural antioxidants, nowadays, synthetic antioxidants, also, have an important place in food and pharmaceutical industries. ${ }^{25}$

In the present study, novel benzothiophene derivatives were synthesized using intramolecular electrophilic cyclization reactions and Palladium catalyzed Sonogashira coupling reactions. Additionally, antimicrobial and antioxidant capacities of new derivatives were also tested to find their potentials as novel drug candidates.

\section{Experimental}

\subsection{General information}

Solvents, reagents, and chemicals used for reactions were purchased from commercial suppliers. Synthesized molecules were analyzed by ${ }^{1} \mathrm{H}$ and ${ }^{13} \mathrm{C}$ NMR. ${ }^{1} \mathrm{H}$ and ${ }^{13} \mathrm{C}$ NMR spectra were recorded on an Agilent NMR $(400 \mathrm{MHz})$ spectrometer. Chemical shifts were reported in parts per million (ppm) downfield from an internal TMS (trimethylsilane) reference. Coupling constants $(J)$ were reported in Hertz $(\mathrm{Hz})$. In addition, spin multiplicities were presented by the following symbols: s (singlet), brs (broad singlet), d (doublet), t (triplet), $\mathrm{q}$ (quartet), and $\mathrm{m}$ (multiplet). DEPT ${ }^{13} \mathrm{C}$ NMR information was given in parentheses as $\mathrm{C}, \mathrm{CH}, \mathrm{CH}_{2}$ and $\mathrm{CH}_{3}$. Flash chromatography was done using thick-walled glass columns and 'flash grade' silica 60 (Merck 230-400 mesh). Thin layer chromatography (TLC) was performed using commercially prepared $0.25 \mathrm{~mm}$ silica gel plates (Silica gel 60, F254) and visualization was effected with short wavelength UV lamp. The relative proportions of a mixture of solvents in chromatography were indicated by volume to volume ratio. All commercially available reagents were used directly without purification unless otherwise stated. All the solvents used in reaction experiments were distilled for purity. The inert atmosphere was created by slight positive pressure (ca. $0.1 \mathrm{psi}$ ) of argon. All reactions were carried out in flame-dried glassware.

\subsection{Synthesis of 2-trimethylsilanylethynylthioanisole}

To a stirred mixture of the 2-iodothioanisole $(2.5 \mathrm{mmol}$, $620 \mathrm{mg})$, THF $(8 \mathrm{~mL})$, ethynyltrimethylsilane $(3 \mathrm{mmol}$, $303 \mathrm{mg}$ ), triethylamine (12.4 mmol, $1.72 \mathrm{~mL}), \mathrm{PdCl}_{2}\left(\mathrm{PPh}_{3}\right)_{2}$ $(0.06 \mathrm{mmol}, 42.1 \mathrm{mg})$ was added $\mathrm{CuI}(0.06 \mathrm{mmol}, 11.4 \mathrm{mg})$ under argon atmosphere. The resulting mixture was stirred at room temperature for $12 \mathrm{~h}$. Then, the mixture was quenched with water $(30 \mathrm{~mL})$, and extracted with $\mathrm{DCM}(3 \times 30 \mathrm{~mL})$. The organic phase was dried over anhydrous $\mathrm{MgSO}_{4}$ and filtered. The solvent was removed under vacuum, and the residue was purified by column chromatography over silica gel with hexane to afford 2-trimethylsilanylethynylthioanisole (5) as light yellow oil in $96 \%$ yield. ${ }^{1} \mathrm{H}$ NMR $\left(400 \mathrm{MHz}, \mathrm{CDCl}_{3}\right) 7.43$ $(\mathrm{dd}, J=7,7,1.5 \mathrm{~Hz} ; 1 \mathrm{H}), 7.27(\mathrm{td}, J=7.5,1.5 \mathrm{~Hz} ; 1 \mathrm{H})$, $7.11(\mathrm{~d}, J=7.4 \mathrm{~Hz} ; 1 \mathrm{H}), 7.05(\mathrm{td}, J=7.5,1.2 \mathrm{~Hz} ; 1 \mathrm{H}), 2.46$ (s, 3H), 0.32 (s, 9H). ${ }^{13} \mathrm{C}$ NMR $\left(100 \mathrm{MHz}, \mathrm{CDCl}_{3}\right) \quad \delta 142.2$, 132.7, 129.1, 124.1, 123.9, 121.1, 102.3, 101.4, 15.0, 0.13; IR (ATR) $v_{\max }\left(\mathrm{cm}^{-1}\right): 3059.5,2980.5,2920.7,2890.6,2154.7$ $(\mathrm{C} \equiv \mathrm{C}), 1435.5,1248.3\left(\mathrm{Si}-\mathrm{CH}_{3}\right), 838.1,747.6,685.2(\mathrm{~S}-$ C). The spectral data were in agreement with those reported previously for this compound. ${ }^{26}$

\subsection{Synthesis of 2-ethynyl thioanisole (6)}

To a stirred mixture of $5(500 \mathrm{mg}, 2.27 \mathrm{mmol})$, methanol $(60 \mathrm{~mL})$, and THF $(20 \mathrm{~mL})$ was added $\mathrm{K}_{2} \mathrm{CO}_{3}(939 \mathrm{mg}$, $6.81 \mathrm{mmol})$. The mixture was stirred at room temperature for $60 \mathrm{~min}$. The reaction mixture extracted with EtOAc $(3 \times$ $15 \mathrm{~mL}$ ). The organic extracts were dried over anhydrous $\mathrm{MgSO}_{4}$ and filtered. The solvent was removed under vacuum, and the residue was purified by column chromatography over silica gel with hexane to afford 2-ethynylthioanisole (6) as yellow oil in $91 \%$ yield: ${ }^{1} \mathrm{H}$ NMR $\left(400 \mathrm{MHz}, \mathrm{CDCl}_{3}\right) \delta 7.46(\mathrm{dd}$, $J=7.6,1.5 \mathrm{~Hz} ; 1 \mathrm{H}), 7.30(\mathrm{td}, J=8.2,1.4 \mathrm{~Hz} ; 1 \mathrm{H}), 7.15(\mathrm{~d}$, $J=8.0 \mathrm{~Hz} ; 1 \mathrm{H}), 7.08(\mathrm{td}, J=8.5,1.0 \mathrm{~Hz} ; 1 \mathrm{H}), 3.5(\mathrm{~s}, 1 \mathrm{H}$, alkyne), 2.48 (s, 3H); ${ }^{13} \mathrm{C} \mathrm{NMR}\left(100 \mathrm{MHz}, \mathrm{CDCl}_{3}\right) \delta 142.0$, 133.1, 129.3, 124.2, 124.1, 120.1, 83.7, 81.0, 60.3; IR (ATR) $v_{\max }\left(\mathrm{cm}^{-1}\right): 3058.32,2982.06,3281.39(\equiv \mathrm{C}-\mathrm{H}), 2920.25$, $2101.87(\mathrm{C} \equiv \mathrm{C}), 1432.71,747.94,614.6(\mathrm{~S}-\mathrm{C})$; HRMS calcd for $\mathrm{C}_{9} \mathrm{H}_{9} \mathrm{~S}, 149.0425[\mathrm{M}+\mathrm{H}]^{+}$found $149.0248[\mathrm{M}+\mathrm{H}]^{+}$. The spectral data were in agreement with those reported previously for this compound. ${ }^{26}$ 
2.4 Synthesis of 2-[(2-(methylthio) phenyl]ethynyl) thiophene 9 via Sonogashira palladium-catalyzed cross-coupling reaction between 2-ethynylthioanisole (6) and 2-bromothiophene (8)

To a solution of 2-bromothiophene $(456.4 \mathrm{mg}, 2.8 \mathrm{mmol})$ in 1 , 4-dioxane (6 mL), $\mathrm{CuI}(32.4 \mathrm{mg}, 0.17 \mathrm{mmol}), \mathrm{PdCl}_{2}(\mathrm{PhCN})_{2}$ $(65.2 \mathrm{mg}, 0.17 \mathrm{mmol})$ were added, followed by $6(3.0 \mathrm{mmol}$, $444 \mathrm{mg})$, diisopropylamine $(1.38 \mathrm{~g}, 13.7 \mathrm{mmol})$, and $\mathrm{P}(t$ $\mathrm{Bu})_{3}(52.9 \mathrm{mg}, 0.33 \mathrm{mmol})$ at room temperature under argon. The mixture was stirred at room temperature for $12 \mathrm{~h}$, and extracted with DCM $(3 \times 20 \mathrm{~mL})$. The organic layer was dried over anhydrous $\mathrm{MgSO}_{4}$ and filtered. The solvent was removed under vacuum, and the residue was purified by column chromatography over silica gel with Hexane/EtOAc (10/1) to afford 2-[(2-(methylthio) phenyl)ethynyl]thiophene (9) as an orange oil in $77 \%$ yield. ${ }^{1} \mathrm{H}$ NMR $(400 \mathrm{MHz}$, $\left.\mathrm{CDCl}_{3}\right) 7.49(\mathrm{~d}, J=7.7 \mathrm{~Hz} ; 1 \mathrm{H}), 7.36-7.30(\mathrm{~m}, 3 \mathrm{H})$, $7.19(\mathrm{~d}, J=8,0 \mathrm{~Hz} ; 1 \mathrm{H}), 7.13(\mathrm{td}, J=8,0,1.1 \mathrm{~Hz} ; 1 \mathrm{H})$, $7.04(\mathrm{~m}, 1 \mathrm{H}), 2.53(\mathrm{~s}, 3 \mathrm{H}) \cdot{ }^{13} \mathrm{C} \mathrm{NMR}\left(100 \mathrm{MHz}, \mathrm{CDCl}_{3}\right) \delta$ $141.8,132.3,132.2,129.1,127.8,127.3,124.4,124.3,123.2$, 121.1, 90.7, 89.2, 15.3; IR (ATR) $v_{\max }\left(\mathrm{cm}^{-1}\right)$ : 3086.06, 2911.91, 2980.6, 2199.72(C $\equiv$ C),1431.12, 753.46, 699.33 (S-C); HRMS calcd for $\mathrm{C}_{13} \mathrm{H}_{11} \mathrm{~S}_{2}, 231.0302[\mathrm{M}+\mathrm{H}]^{+}$found $231.0313[\mathrm{M}+\mathrm{H}]^{+}$. The spectral data were in agreement with those reported previously for this compound. ${ }^{27}$

\subsection{Synthesis of 2-[(2-(methylthio) phenyl) ethynyl]} thiophene 9 via Sonogashira palladium-catalyzed cross-coupling reaction between 2-ethynylthioanisole (6) and 2-iodothiophene (7)

To a mixture of 2-iodothiophene $(707 \mathrm{mg}, 3.37 \mathrm{mmol}), 6$ (500 mg, $3.37 \mathrm{mmol}), \mathrm{DMF}$ (6 mL), $\mathrm{PdCl}_{2}\left(\mathrm{PPh}_{3}\right)_{2}$ (118.3 mg, $0.17 \mathrm{mmol})$, and triethylamine $(1.9 \mathrm{~mL}, 13.7 \mathrm{mmol})$ at $0{ }^{\circ} \mathrm{C}$ under argon gas was added $\mathrm{CuI}(32.1 \mathrm{mg}, 0.17 \mathrm{mmol})$. The mixture was stirred at room temperature for $4 \mathrm{~h}$ and extracted with ether $(3 \times 20 \mathrm{~mL})$. The organic layer was dried over anhydrous $\mathrm{MgSO}_{4}$ and filtered. The solvent was removed under vacuum, and the residue was purified by column chromatography over silica gel with Hexane/EtOAc (10/1) to afford compound 9 as orange oil in $\mathbf{7 1 \%}$ yield.

\subsection{Synthesis of 3-iodo-2-(thiophen-2-yl) benzo[b] thiophene (10) via electrophilic cyclization reaction}

To a solution of 2-[(2-(methylthio phenyl) ethynyl] thiophene (9) $(230 \mathrm{mg}, 1 \mathrm{mmol})$ in $\mathrm{CH}_{2} \mathrm{Cl}_{2}(10 \mathrm{~mL})$ was added $\mathrm{I}_{2}(762 \mathrm{mg}, 3 \mathrm{mmol})$ at room temperature. After stirring for $30 \mathrm{~min}$, the saturated aqueous solution of $\mathrm{Na}_{2} \mathrm{~S}_{2} \mathrm{O}_{3}$ was added into the reaction mixture and extracted with EtOAc $(3 \times 20 \mathrm{~mL})$. The combined organic phase was dried over anhydrous $\mathrm{MgSO}_{4}$ and filtered. The solvent was removed under vacuum, and the residue was purified by column chromatography over silica gel with hexane/EtOac (19/1) to afford 3-iodo-2-(thiophen-2-yl) benzo[b]thiophene (10) as light yellow solid in $99 \%$ yield. ${ }^{1} \mathrm{H}$ NMR $\left(400 \mathrm{MHz}, \mathrm{CDCl}_{3}\right)$ $7.82(\mathrm{~d}, J=8.1 \mathrm{~Hz} ; 1 \mathrm{H}), 7.75(\mathrm{~d}, J=9.1 \mathrm{~Hz} ; 1 \mathrm{H}), 7.62$ $(\mathrm{d} . \mathrm{d}, J=4,0,1.1 \mathrm{~Hz} ; 1 \mathrm{H}), 7.48-7.44(\mathrm{~m}, 2 \mathrm{H}), 7.39(\mathrm{td}$, $J=8,0,1.2 \mathrm{~Hz} ; 1 \mathrm{H}), 7.17(\mathrm{~m}, 1 \mathrm{H}) .{ }^{13} \mathrm{C} \mathrm{NMR}(100 \mathrm{MHz}$, $\left.\mathrm{CDCl}_{3}\right) \delta 142.4,138.1,136.0,135.9,128.8,127.5,127.4$, 126.4, 125.9, 125.8, 122.0, 79.5; IR (ATR) $v_{\max }\left(\mathrm{cm}^{-1}\right)$ : 698.75, 815.0, 1419.47, 2912.23, 2980.61, 3085.91; HRMS calcd for $\mathrm{C}_{12} \mathrm{H}_{7} \mathrm{~S}_{2}, 341.9034$ found; 341.9059 .

\subsection{General procedure of Sonogashira cross- coupling reaction between 3-iodo-2-(thiophen-2-yl) benzo[b]thiophene (10) and alkynes}

To a stirred mixture of 3-iodo-2-(thiophen-2-yl) benzo[b] thiophene $10(0.7 \mathrm{mmol}, 239.5 \mathrm{mg})$, dimethylformamide (DMF) $(7.5 \mathrm{~mL})$, alkyne $(0.75 \mathrm{mmol})$, triethylamine $(3.0 \mathrm{~mL})$, $\mathrm{PdCl}_{2}\left(\mathrm{PPh}_{3}\right)_{2}(0.035 \mathrm{mmol}, 24.5 \mathrm{mg})$ under argon atmosphere was added $\mathrm{CuI}(0.035 \mathrm{mmol}, 6.6 \mathrm{mg})$. The resulting mixture was stirred at room temperature for $12 \mathrm{~h}$. It was quenched with water $(30 \mathrm{~mL})$ and extracted with DCM $(3 \times$ $30 \mathrm{~mL}$ ). The organic layer was dried over anhydrous $\mathrm{MgSO}_{4}$ and filtered. The solvent was removed under vacuum, and the residue was purified by column chromatography over silica gel with Hexane/EtOAc (19/1) to afford compounds (12A-L).

\section{3-(Phenylethynyl)-2-(thiophen-2-yl)benzo $[b]$ thiophene} (12A) was isolated in $\mathbf{9 4 \%}$ yield as a green solid. M.p: 85.1-86.7 ${ }^{\circ} \mathrm{C} ;{ }^{1} \mathrm{H}$ NMR $\left(400 \mathrm{MHz}, \mathrm{CDCl}_{3}\right) \delta 7.99(\mathrm{~d}$, $J=7.4 \mathrm{~Hz} ; 1 \mathrm{H}), 7.77(\mathrm{~d}, J=8.0 \mathrm{~Hz} ; 1 \mathrm{H}), 7.74$ $7.70(\mathrm{~m}, 2 \mathrm{H}), 7.66(\mathrm{~d}, J=3.72 \mathrm{~Hz} ; 1 \mathrm{H}), 7.49-7.37(\mathrm{~m}$, $6 \mathrm{H}), 7.13-7.15(\mathrm{~m}, 1 \mathrm{H}) .{ }^{13} \mathrm{C} \mathrm{NMR}\left(100 \mathrm{MHz}, \mathrm{CDCl}_{3}\right)$ $\delta 140.8 ; 140.2,137.0,136.5,131.8,128.8,128.7,127.5$, 127.2, 127.16, 125.7, 125.3, 123.5, 123.3, 122.2, 113.0, 97.9, 84.2; IR (ATR) $v_{\max }\left(\mathrm{cm}^{-1}\right): 3062.96,2980.48,2922.72$, 2199.68, 1438.03, 1237.22, 746.05, 683.58; HRMS: calcd for $\mathrm{C}_{20} \mathrm{H}_{13} \mathrm{~S}_{2}, 317.0458[\mathrm{M}+\mathrm{H}]^{+}$found, $317.0457[\mathrm{M}+\mathrm{H}]^{+}$.

\section{3-(p-Tolylethynyl)-2-(thiophen-2-yl)benzo[b]thiophene} (12B) was isolated in $\mathbf{6 5 \%}$ yield as an yellow solid. M.p.: 120.0-121.5 ${ }^{\circ} \mathrm{C} ;{ }^{1} \mathrm{H}$ NMR $\left(400 \mathrm{MHz}, \mathrm{CDCl}_{3}\right) \delta 7.98(\mathrm{~d}, J=$ $8.0 \mathrm{~Hz} ; 1 \mathrm{H}), 7.76(\mathrm{~d}, J=7.9 \mathrm{~Hz} ; 1 \mathrm{H}), 7.66(\mathrm{~d}, J=3.8 \mathrm{~Hz}$; $1 \mathrm{H}), 7.60(\mathrm{~d}, J=8.0 \mathrm{~Hz} ; 2 \mathrm{H}), 7.48-7.36(\mathrm{~m}, 3 \mathrm{H}), 7.24(\mathrm{~d}$, $J=7.9 \mathrm{~Hz} ; 2 \mathrm{H}), 7.15-7.12(\mathrm{~m}, 1 \mathrm{H}) .{ }^{13} \mathrm{C} \mathrm{NMR}(100 \mathrm{MHz}$, $\left.\mathrm{CDCl}_{3}\right) \delta 140.8,139.8,139.0,137.0,136.6,131.7,129.5$, $127.4,127.04,127.01,125.6,125.2,123.3,122.1,120.4$, 113.1, 98.2, 83.6, 21.8; IR (ATR) $v_{\max }\left(\mathrm{cm}^{-1}\right): 3068.87$, 2980.59, 2918.12, 1437.45, 806.73, 752.44; HRMS:calcd for $\mathrm{C}_{21} \mathrm{H}_{15} \mathrm{~S}_{2}, 331.0615[\mathrm{M}+\mathrm{H}]^{+}$found $331.0699[\mathrm{M}+\mathrm{H}]^{+}$.

\section{3-(Hept-1-yn-1-yl)-2-(thiophen-2-yl)benzo[b]thiophene} (12C) was isolated in $\mathbf{8 2 \%}$ yield as a green solid. ${ }^{1} \mathrm{H}$ NMR $\left(400 \mathrm{MHz}, \mathrm{CDCl}_{3}\right) \delta 7.90(\mathrm{~d}, J=8.0 \mathrm{~Hz} ; 1 \mathrm{H}), 7.74(\mathrm{~d}, J=$ $1.08 \mathrm{~Hz} ; 1 \mathrm{H}), 7.63(\mathrm{~d}, J=4.7 \mathrm{~Hz} ; 1 \mathrm{H}), 7.45-7.36(\mathrm{~m}, 3 \mathrm{H})$, $7.14-7.10(\mathrm{~m}, 1 \mathrm{H}), 2.65(\mathrm{t}, J=7.1 \mathrm{~Hz} ; 2 \mathrm{H}), 1.82-1.74$ $(\mathrm{m}, 2 \mathrm{H}), 1.61-1.53(\mathrm{~m}, 2 \mathrm{H}), 1.48-1.40(\mathrm{~m}, 2 \mathrm{H}), 0.98(\mathrm{t}, J=$ $7.3 \mathrm{~Hz} ; 3 \mathrm{H}) .{ }^{13} \mathrm{C} \mathrm{NMR}\left(100 \mathrm{MHz}, \mathrm{CDCl}_{3}\right) \delta 141.3,138.8$, 137.0, 136.7, 127.3, 126.7, 126.5, 125.5, 125.1, 123.3, 122.1, $113.8,99.8$, 75.2, 31.5, 30.0, 28.5, 22.5, 20.3, 14.3; IR ( $\mathrm{KBr}$, thin film) $\mathrm{v}_{\max }\left(\mathrm{cm}^{-1}\right): 3067.19,2950.81,2929.27,2855.59$, 
2215.36 ( $\mathrm{C} \equiv \mathrm{C}), 1455.44,1420.67,836.14,698.05$; HRMS calcd. for $\mathrm{C}_{19} \mathrm{H}_{19} \mathrm{~S}_{2}, 311.0850[\mathrm{M}+\mathrm{H}]^{+}$; found 311.0843 $[\mathrm{M}+\mathrm{H}]^{+}$.

3-[(3-Bromothiophen-2-yl)ethynyl)-2-(thiophen-2-yl]benzo[b]thiophene (12D) was isolated in $71 \%$ yield as an yellow solid. M.p.: 91.7-62.6 ${ }^{\circ} \mathrm{C} ;{ }^{1} \mathrm{H}$ NMR (400 $\mathrm{MHz} \mathrm{CDCl}_{3}$ ) $\delta 8.03(\mathrm{~d}, J=7.4 \mathrm{~Hz} ; 1 \mathrm{H}), 7.78-7.72(\mathrm{~m}, 2 \mathrm{H}), 7.50-7.34$ $(\mathrm{m}, 3 \mathrm{H}), 7.31(\mathrm{~d}, J=5.3 \mathrm{~Hz}, 1 \mathrm{H}), 7.14-7.13(\mathrm{~m}, 1 \mathrm{H})$, $7.06(\mathrm{~d}, J=5.4,1 \mathrm{H}) \cdot{ }^{13} \mathrm{C} \mathrm{NMR}\left(100 \mathrm{MHz}, \mathrm{CDCl}_{3}\right) \delta 140.8$, 140.6, 137.0, 136.3, 130.6, 127.9, 127.7, 127.4, 127.3, 125.8, $125.5,123.4,122.2,121.2,116.4,112.3,91.8,89.3$; IR (ATR) $\mathrm{V}_{\max }\left(\mathrm{cm}^{-1}\right): 3099.53,3054.27,2980.48,2922.74,2195.37$ $(\mathrm{C} \equiv \mathrm{C}), 1436.51,1149.67,862.56$, 688.91; HRMS calcd for $\mathrm{C}_{18} \mathrm{H}_{10} \mathrm{~S}_{3} \mathrm{Br}, 400.9128[\mathrm{M}+\mathrm{H}]^{+}$found $400.9131[\mathrm{M}+\mathrm{H}]^{+}$.

3-(4-Aminobenzoethynyl)-2-(thiophen-2-yl) benzo[b]thiophene (12E) was isolated in $\mathbf{9 2 \%}$ yield as a green solid. ${ }^{1} \mathrm{H}$ NMR $\left(400 \mathrm{MHz}, \mathrm{CDCl}_{3}\right) \delta 7.98(\mathrm{~d}, J=10.0 \mathrm{~Hz} ; 1 \mathrm{H})$, $7.76(\mathrm{~d}, J=9.6 \mathrm{~Hz} ; 1 \mathrm{H}), 7.65(\mathrm{~d} . \mathrm{d}, J=3.72 \mathrm{~Hz}, 1.12 \mathrm{~Hz}$; $1 \mathrm{H}), 7.51(\mathrm{~d}, J=8.7 \mathrm{~Hz} ; 2 \mathrm{H}), 7.47-7.35(\mathrm{~m}, 3 \mathrm{H}), 7.14-$ $7.11(\mathrm{~m}, 1 \mathrm{H}), 6.70(\mathrm{~d}, J=8.7 \mathrm{~Hz} ; 2 \mathrm{H}), 3.9$ (brs, 2H). ${ }^{13} \mathrm{C} \mathrm{NMR}\left(100 \mathrm{MHz}, \mathrm{CDCl}_{3}\right) \delta 147.2,140.9,138.8,136.9$, 136.8, 133.2, 127.4, 126.8 (including 2C), 125.6, 125.2, 123.4, 122.1, 115.1, 113.6, 112.8, 98.9, 82.2; IR (ATR) $v_{\max }\left(\mathrm{cm}^{-1}\right): 3648.08,3378.68,2955.73,2922.29,2193.60$, 1603.81, 1613.73, 1288.05, 1174.35, 906.16, 825.42, 728.67, 696.05; HRMS:calcd for $\mathrm{C}_{20} \mathrm{H}_{14} \mathrm{~S}_{2} \mathrm{~N}, 332.0568[\mathrm{M}+\mathrm{H}]^{+}$ found $332.0559[\mathrm{M}+\mathrm{H}]^{+}$.

\section{3-(4-Methoxybenzoethynyl)-2-(thiophen-2-yl) benzo[b]} thiophene $(\mathbf{1 2 F})$ was isolated in $\mathbf{7 0 \%}$ yield as a light green solid. M.p.: 99.5-100.8 ${ }^{\circ} \mathrm{C} ;{ }^{1} \mathrm{H}$ NMR $\left(400 \mathrm{MHz}, \mathrm{CDCl}_{3}\right) \delta$ $7.98(\mathrm{~d}, J=10.2 \mathrm{~Hz} ; 1 \mathrm{H}), 7.76(\mathrm{~d}, J=7.9 \mathrm{~Hz}, 1 \mathrm{H}), 7.66-$ $7.63(\mathrm{~m}, 3 \mathrm{H}), 7.45-7.351(\mathrm{~m}, 3 \mathrm{H}), 7.14-7.11(\mathrm{~m}, 1 \mathrm{H}), 6.96$ $(\mathrm{d}, J=14.4 \mathrm{~Hz}, 2 \mathrm{H}) 3.87(\mathrm{~s}, 3 \mathrm{H}) .{ }^{13} \mathrm{C} \mathrm{NMR}(100 \mathrm{MHz}$, $\left.\mathrm{CDCl}_{3}\right) \delta 160.0,140.8,139.4,136.9,136.7,133.3,127.4$, 127.0, 126.9, 125.6, 125.2, 123.4, 122.9, 115.6, 114.4, 113.3, 98.1, 82.9, 55.6; IR (ATR) $v_{\max }\left(\mathrm{cm}^{-1}\right): 3099.03,3050.45$, $2969.85,2836.00,2537.06,2198.92,1603.21,1455.42$, 1290.88, 1248.09, 1169.13, 1022.60, 834.25, 753.21, 689.80; HRMS: calcd for $\mathrm{C}_{21} \mathrm{H}_{14} \mathrm{OS}_{2}, 346.0486$, found 346.0488.

3-(2,5-Dimethylbenzoethynyl)-2-(thiophen-2-yl) benzo[b] thiophene (12G) was isolated in $69 \%$ yield as an orange solid. M.p.: $124.7-126.2{ }^{\circ} \mathrm{C} ;{ }^{1} \mathrm{H}$ NMR $\left(400 \mathrm{MHz}, \mathrm{CDCl}_{3}\right) \delta$ $8.0(\mathrm{~d}, J=6.0 \mathrm{~Hz} ; 1 \mathrm{H}), 7.8(\mathrm{~d}, J=9.6 \mathrm{~Hz} ; 1 \mathrm{H}), 7.69(\mathrm{dd}$, $J=4.0 \mathrm{~Hz}, 1.2 \mathrm{~Hz} ; 1 \mathrm{H}), 7.52(\mathrm{~s}, 1 \mathrm{H}), 7.50-7.35$ (m, 3H), 7.20 $(\mathrm{d}, J=7.8 \mathrm{~Hz} ; 1 \mathrm{H}), 7.16-7.12(\mathrm{~m}, 2 \mathrm{H}), 2.65(\mathrm{~s}, 3 \mathrm{H}), 2.40$ (s, 3H). ${ }^{13} \mathrm{C}$ NMR $\left(100 \mathrm{MHz}, \mathrm{CDCl}_{3}\right) \delta 140.9,139.5,137.2$, 137.0, 136.5, 135.4, 132.7, 129.8, 129.7, 127.4, 127.1, 127.0, $125.6,125.3,123.3,123.1,122.2,113.4$, 97.1, 87.4, 21.1, 20.9; IR (ATR) $\mathrm{v}_{\max }\left(\mathrm{cm}^{-1}\right): 3058.44,2980.45,2915.37$, 2727.39, 2190.46, 1936.28, 1901.14, 1373.30, 1420.57, 1228.57, 813.75, 695.58, 573.38; HRMS calcd for $\mathrm{C}_{22} \mathrm{H}_{17} \mathrm{~S}_{2}$, $345.0772[\mathrm{M}+\mathrm{H}]^{+}$found $345.0768[\mathrm{M}+\mathrm{H}]^{+}$. .

3-(2-Naphthylethynyl)-2-(thiophen-2-yl) benzo[b]thiophene $\mathbf{( 1 2 H )}$ was isolated in $\mathbf{9 3 \%}$ yield as an yellow solid. M.p.: $140.3-143.3{ }^{\circ} \mathrm{C} ;{ }^{1} \mathrm{H}$ NMR $\left(400 \mathrm{MHz}, \mathrm{CDCl}_{3}\right) \delta 8.6$ $(\mathrm{d}, J=8.5 \mathrm{~Hz} ; 1 \mathrm{H}), 8.1(\mathrm{~d}, J=8.0 \mathrm{~Hz} ; 1 \mathrm{H}), 7.97-7.91(\mathrm{~m}$, $3 \mathrm{H}), 7.8(\mathrm{~d}, J=9.0 \mathrm{~Hz} ; 1 \mathrm{H}), 7.22(\mathrm{dd}, J=4.0,1.2 \mathrm{~Hz}$; 1H), 7.68-7.64 (m, 1H), 7.60-7.48 (m, 3H), 7.44-7.40 (m, 2H), 7.16-7.12 (m, 1H). ${ }^{13} \mathrm{C}$ NMR $\left(100 \mathrm{MHz}, \mathrm{CDCl}_{3}\right) \delta$ $140.9,140.2$, 137.1, 136.5, 133.5, 133.3, 131.0, 129.3, 128.6, $127.5,127.3,127.25,127.22,126.8,126.6,125.7,125.6$, $125.5,123.4,122.2,121.2,113.2,96.1,88.8$; IR (ATR) $\mathrm{V}_{\max }\left(\mathrm{cm}^{-1}\right): 3051.37,2918.28,2191.60,1936.73,1727.62$, 1388.38, 791.93, 768.18, 694.03; HRMS calcd for 367.0615 $\mathrm{C}_{24} \mathrm{H}_{15} \mathrm{~S}_{2}[\mathrm{M}+\mathrm{H}]^{+}$found $367.0602[\mathrm{M}+\mathrm{H}]^{+}$.

3-(2-Thioanisolethynyl)-2-(thiophen-2-yl) benzo[b]thiophene (12I) was isolated in $\mathbf{8 1 \%}$ yield as a yellow solid. M.p.: $165.3-167.2{ }^{\circ} \mathrm{C} ;{ }^{1} \mathrm{H}$ NMR $\left(400 \mathrm{MHz} \mathrm{CDCl}_{3}\right) \delta 8.16$ $(\mathrm{d}, J=8.0 \mathrm{~Hz} ; 1 \mathrm{H}), 7.75(\mathrm{~d}, J=7.96 \mathrm{~Hz} ; 1 \mathrm{H}) 7.72$ $(\mathrm{dd}, J=3.7 \mathrm{~Hz}, 1.08 \mathrm{~Hz} ; 1 \mathrm{H}), 7.66(\mathrm{dd}, J=7.7 \mathrm{~Hz}$, $1.32 \mathrm{~Hz} ; 1 \mathrm{H}), 7.49-7.33(\mathrm{~m}, 4 \mathrm{H})$ 7.27-7.24 (m, 1H), 7-22$711(\mathrm{~m}, 2 \mathrm{H}), 2.56(\mathrm{~s}, 3 \mathrm{H}) ;{ }^{13} \mathrm{C} \mathrm{NMR}\left(100 \mathrm{MHz}, \mathrm{CDCl}_{3}\right) \delta$ $141.7,141.0,136.9,136.5,132.64,130.0,129.2,127.6,127.3$, 127.2, 125.7, 125.5, 124.6, 124.5, 123.8, 122.1, 121.7, 113.0, 95.1, 90.5, 15.52; IR (ATR) $v_{\max }\left(\mathrm{cm}^{-1}\right): 3054.12,2919.23$, 2196.83, 1433.38, 1069.32, 745.44, 691.63; HRMS: calcd for $\mathrm{C}_{21} \mathrm{H}_{15} \mathrm{~S}_{3}, 363.0336[\mathrm{M}+\mathrm{H}]^{+}$found $363.0335[\mathrm{M}+\mathrm{H}]^{+}$.

3-(2-Aminobenzoethynyl)-2-(thiophen-2-yl) benzo[ $b]$ thiophene (12J) was isolated in $\mathbf{9 1 \%}$ yield as an yellow-green solid. M. p.: 89.9-94.5 ${ }^{\circ} \mathrm{C} ;{ }^{1} \mathrm{H}$ NMR (400 MHz, $\left.\mathrm{CDCl}_{3}\right)$ $\delta 7.98-7.96(\mathrm{~d}, J=8.0,1 \mathrm{H}), 7.78-7.76(\mathrm{~d}, J=8.0$, $1 \mathrm{H}), 7.66-7.64(\mathrm{~m}, 1 \mathrm{H}), 7.56-7.54(\mathrm{~m}, 1 \mathrm{H}), 7.48-7.37(\mathrm{~m}$, $3 \mathrm{H}), 7.24-7.20(\mathrm{~m}, 1 \mathrm{H}), 7.13-7.11(\mathrm{~m}, 1 \mathrm{H}), 6.83-6.78(\mathrm{~m}$, 2H), 4.47 (brs, $2 \mathrm{H}) ;{ }^{13} \mathrm{C} \mathrm{NMR}\left(100 \mathrm{MHz}, \mathrm{CDCl}_{3}\right) \delta 148$, 140.7, 139.5, 137.0, 136.4, 132.6, 130.4, 127.5, 127.1, 127.0, 125.7, 125.4, 123.2, 122.2, 118.3, 114.7, 113.1, 108.1, 94.68, 89.0; IR (ATR) $\mathrm{v}_{\max }\left(\mathrm{cm}^{-1}\right): 3646.46,3388.01,3291.45$, 2980.39, 2921.93, 2190.03, 1727.63, 1642.99, 1620.79, 1437.80, 1252.95, 1153.55, 753.99, 694.32; HRMS calcd for $\mathrm{C}_{20} \mathrm{H}_{14} \mathrm{NS}_{2} 332.0567[\mathrm{M}+\mathrm{H}]^{+}$found $332.0568[\mathrm{M}+\mathrm{H}]^{+}$.

3-(Trimethylsilylethynyl)-2-(thiophen-2-yl) benzo[b]thiophene (12K) was isolated in $\mathbf{8 9 \%}$ yield as a red oil. ${ }^{1} \mathrm{H}$ NMR (400 MHz, $\left.\mathrm{CDCl}_{3}\right) \delta 7.90(\mathrm{~d}, J=10,0 \mathrm{~Hz} ; 1 \mathrm{H}), 7.74$ $(\mathrm{d}, J=8.0 \mathrm{~Hz} ; 1 \mathrm{H}), 7.68(\mathrm{~d}, J=4,0 \mathrm{~Hz} ; 1 \mathrm{H}), 7.47-7.41(\mathrm{~m}$, $2 \mathrm{H}), 7.36(\mathrm{td}, J=8.0,0.9 \mathrm{~Hz} ; 1 \mathrm{H}), 7.14-7.11(\mathrm{~m}, 1 \mathrm{H}), 0.40$ $(\mathrm{s}, 9 \mathrm{H}) ;{ }^{13} \mathrm{C} \mathrm{NMR}\left(100 \mathrm{MHz}, \mathrm{CDCl}_{3}\right) \delta 141.1,140.9,136.8$, 136.4, 127.4, 127.2, 127.0, 125.6, 125.3, 123.4, 122.0, 112.9, 104.1, 99.2, 0.13; IR (ATR) $v_{\max }\left(\mathrm{cm}^{-1}\right): 2958.21,2148.01$, 1438.15, 1247.46, 908.99, 839.30, 756.33, 696.03; HRMS cald for $\mathrm{C}_{17} \mathrm{H}_{17} \mathrm{~S}_{2} \mathrm{Si}, 313.0541[\mathrm{M}+\mathrm{H}]^{+}$found; 313.0537 $[\mathrm{M}+\mathrm{H}]^{+}$.

\subsection{General procedure of desilylation reaction for the} synthesis of 3-ethynyl-2-(thiophen-2-yl) benzo[b] thiophene (12L)

To a solution of 3-(trimethylsilylethynyl)-2-(thiophen-2-yl) benzo [b] thiophene (12l) $(56 \mathrm{mg}, 0.17 \mathrm{mmol})$ in methanol $(15 \mathrm{~mL})$ and THF $(5 \mathrm{~mL})$ was added $\mathrm{K}_{2} \mathrm{CO}_{3}(74.3 \mathrm{mg}, 0.53$ $\mathrm{mmol})$ at room temperature for $1 \mathrm{~h}$. Then, organic solvents were removed under reduced pressure and the residue was 
extracted with DCM $(10 \mathrm{~mL} \times 3)$. The solvent was removed under vacuum, and the residue was purified by column chromatography over silica gel with Hexane/EtOAc (19/1) to afford 3-ethynyl-2-(thiophen-2-yl) benzo [b] thiophene $(\mathbf{1 2 L})$ as a red oil in $\mathbf{8 3 \%}$ yield. ${ }^{1} \mathrm{H}$ NMR $\left(400 \mathrm{MHz}, \mathrm{CDCl}_{3}\right)$ $\delta 7.91(\mathrm{~d}, J=8,0 \mathrm{~Hz} ; 1 \mathrm{H}), 7.77-7.70(\mathrm{~m}, 2 \mathrm{H}), 7.48-$ $7.34(\mathrm{~m}, 3 \mathrm{H}), 7.16-7.10(\mathrm{~m}, 1 \mathrm{H}), 3.70(\mathrm{~s}, 1 \mathrm{H}) ;{ }^{13} \mathrm{C} \mathrm{NMR}$ $\left(100 \mathrm{MHz}_{2} \mathrm{CDCl}_{3}\right) \delta 141.4,140.1,136.9,136.0,127.7,127.4$, $127.1,125.7,125.4,123.2,122.1,111.8,85.8,78.3$; IR (ATR) $v_{\max }\left(\mathrm{cm}^{-1}\right): 3286.03,3101.49,2922.12,2851.51,2095.61$, 1940.45, 1791.21, 1437.68, 1188.59, 752.07, 690.36, 647.28, 584.34; HRMS cald for $\mathrm{C}_{14} \mathrm{H}_{9} \mathrm{~S}_{2}, 241.0146[\mathrm{M}+\mathrm{H}]^{+}$found; $241.0139[\mathrm{M}+\mathrm{H}]^{+}$.

\subsection{Synthesis of 3-(3-iodobenzo[b]thiophen-2-yl)-2- (thiophen-2-yl) benzo [b] thiophene (14)}

To a solution of the 3-(2-thioanisolethynyl)-2-(thiophen-2yl) benzo $[b]$ thiophene $12 \mathrm{I}(85 \mathrm{mg}, 0.23 \mathrm{mmol})$ in dichloromethane $(5 \mathrm{~mL})$ was added $\mathrm{I}_{2}(173.8 \mathrm{mg}, 0.69 \mathrm{mmol})$ under argon atmosphere. The mixture was stirred at room temperature for $1 \mathrm{~h}$. It was quenched with sat. sodium thiosulfate $(30 \mathrm{~mL})$, and extracted with DCM $(3 \times 20 \mathrm{~mL})$. The organic layer was dried over anhydrous $\mathrm{MgSO}_{4}$ and filtered. The solvent was removed under vacuum, and the residue was purified by column chromatography over silica gel with Hexane to afforded 3-(3-iodobenzo[b]thiophen-2-yl)-2-(thiophen-2yl) benzo[b]thiophene (14) as a light yellow solid in $\mathbf{8 1 \%}$ yield. ${ }^{1} \mathrm{H}$ NMR $\left(400 \mathrm{MHz}, \mathrm{CDCl}_{3}\right) \delta 7.90-7.85(\mathrm{~m}, 3 \mathrm{H})$, 7.57-7.47 (m, 2H), 7.42-7.33 (m, 3H), 7.28-7.27 (m, 1H), 7.24-7.22 (m, 1H), 6.99-6.97 (m, 1H); ${ }^{13} \mathrm{C} \mathrm{NMR} \mathrm{(100} \mathrm{MHz,}$ $\left.\mathrm{CDCl}_{3}\right) \delta 141.5,140.8,140.5,137.8,137.7,136.3,135.5$, $128.0,127.5,127.4,126.4,126.1,125.6,125.4,125.3,124.8$, 123.4, 122.8, 122.2, 86.48; IR (ATR) $v_{\max }\left(\mathrm{cm}^{-1}\right): 3104.56$, 3057.09, 2930.00, 2851.61, 1728.33, 1430.00, 1244.14, 753.26, 703.55; HRMS calcd for $\mathrm{C}_{20} \mathrm{H}_{11} \mathrm{IS}_{3}, 473.9067$; found 473.9072 .

\subsection{General procedure for the synthesis of 3-} (arylethynyl)-2'-(thiophen-2-yl)-2,3'-bibenzo[b] thiophene (15)

To a stirred mixture of $\mathbf{1 4}(0.7 \mathrm{mmol})$, DMF $(7.5 \mathrm{~mL})$, alkyne $(0.75 \mathrm{mmol})$, triethylamine $(3.0 \mathrm{~mL}), \mathrm{PdCl}_{2}\left(\mathrm{PPh}_{3}\right)_{2}$ $(0.035 \mathrm{mmol})$ under argon gas was added $\mathrm{CuI}(0.035 \mathrm{mmol}$, $6.7 \mathrm{mg}$ ). The resulting mixture was stirred at room temperature for $12 \mathrm{~h}$ Water $(30 \mathrm{~mL})$ was added to the reaction mixture, and the resulting solution was extracted with DCM $(3 \times 30 \mathrm{~mL})$. The organic layer was dried over anhydrous $\mathrm{MgSO}_{4}$ and filtered. The solvent was removed under vacuum, and the residue was purified by column chromatography over silica gel with Hexane/EtOAc (19/1) to afford compounds (15A-D).

\section{3-(3-(p-Tolylethynyl)benzo[b]thiophen-2-yl)-2-(thioph-} en-2-yl)benzo[b]thiophene $15 \mathrm{~A}$ was isolated in $\mathbf{8 9 \%}$ yield as a yellow solid : M.p.: $139.7-141.2{ }^{\circ} \mathrm{C} ;{ }^{1} \mathrm{H} \mathrm{NMR}(400 \mathrm{MHz}$, CDCl3) $\delta$ 8.08-8.10 (d, $J=8.0,1 \mathrm{H}), 7.88-7.90(\mathrm{~d}, J=$
8.0, 1H), 7.84-7.86 (m, 1H), 7.66-7.69 (m, 1H), 7.45-7.55 $(\mathrm{m}, 2 \mathrm{H}), 7.34-7.40(\mathrm{~m}, 2 \mathrm{H}), 7.23-7.26(\mathrm{~m}, 2 \mathrm{H}), 7.11-7.13$ $(\mathrm{d}, J=8.0,2 \mathrm{H}), 7.03-7.05(\mathrm{~d}, J=8.0,2 \mathrm{H}), 6.94-6.97$ $(\mathrm{m}, 1 \mathrm{H}), 2.31(\mathrm{~s}, 3 \mathrm{H}) ;{ }^{13} \mathrm{C} \mathrm{NMR}\left(100 \mathrm{MHz}, \mathrm{CDCl}_{3}\right) \delta$ 140.9, 139.9, 139.8, 138.9, 138.6, 138.0, 137.2, 135.8, 131.7, $129.1,127.68,127.66,127.4,125.6,125.2,125.1,125.0$, 124.1, 123.8 (include 2C), 122.7, 122.1, 120.2, 120.1, 95.4, 82.2, 21.7; IR (ATR) $v_{\max }\left(\mathrm{cm}^{-1}\right):$ 698.50, 728.72, 755.38, 814.32, 1435.36, 2205.17, 2853.61, 2920.76; HRMS calcd for $\mathrm{C}_{29} \mathrm{H}_{19} \mathrm{~S}_{3}, 463.0649[\mathrm{M}+\mathrm{H}]^{+;}$found $463.0636[\mathrm{M}+\mathrm{H}]^{+}$.

3-(3-(2,5-Dimethylbenzoethynyl)benzo[b]thiophen-2-yl)2-(thiophen-2-yl)benzo[b]thiophene 15B was isolated in 90\% yield as a yellow solid: ${ }^{1} \mathrm{H}$ NMR $\left(400 \mathrm{MHz}, \mathrm{CDCl}_{3}\right)$ $\delta 8.12-8.14(\mathrm{~d}, J=8.0,1 \mathrm{H}), 7.90-7.92(\mathrm{~d}, J=8.0,1 \mathrm{H})$, 7.83-7.85 (m, 1H), 7.54-7.59 (m, 2H), 7.47-7.51 (m, 1H), 7.31-7.39 (m, 2H), 7.26-7.28 (m, 1H), 7.21-7.22 (m, 1H), $7.10(\mathrm{~s}, 1 \mathrm{H}), 6.94-6.96(\mathrm{~m}, 3 \mathrm{H}), 2.24(\mathrm{~s}, 3 \mathrm{H}), 1.90(\mathrm{~s}, 3 \mathrm{H}) ; 13 \mathrm{C}$ NMR $\left(100 \mathrm{MHz}, \mathrm{CDCl}_{3}\right) \delta 141.0,140.0,139.8,138.9,137.8$, 137.4, 137.3, 135.7, 135.0, 132.4, 129.4, 129.38, 127.7, 127.5, $127.3,125.7,125.24,125.16,125.1,124.0,123.8,123.7$, $122.8,122.7,122.0,120.7,94.3,86.3,20.9,19.8$; IR (ATR) $\operatorname{vmax}\left(\mathrm{cm}^{-1}\right):$ 661.07, 729.36, 756.18, 1030.58, 1232.42, 1435.79, 1456.13, 1733.94, 2202.29, 2922.48; HRMS calcd for $\mathrm{C}_{30} \mathrm{H}_{21} \mathrm{~S}_{3}, 477.0805[\mathrm{M}+\mathrm{H}]^{+}$; found $477.0770[\mathrm{M}+\mathrm{H}]^{+}$.

3-(3-(2-Naphthylethynyl)benzo[b]thiophen-2-yl)-2-(thiophen-2-yl)benzo[b]thiophene $15 \mathrm{C}$ was isolated in $\mathbf{7 6 \%}$ yield as an yellow solid: M.p.: $153.2-154.7{ }^{\circ} \mathrm{C} ;{ }^{1} \mathrm{H}$ NMR $\left(400 \mathrm{MHz}, \mathrm{CDCl}_{3}\right) \delta 8.21-8.23(\mathrm{~d}, J=8.0,1 \mathrm{H}), 7.90$ $7.95(\mathrm{~m}, 2 \mathrm{H}), 7.73-7.76(\mathrm{~m}, 2 \mathrm{H}), 7.66-7.68(\mathrm{~d}, J=8.0$, 1H), 7.50-7.61 (m, 4H), 7.34-7.44 (m, 4H), 7.30-7.31 (m, $1 \mathrm{H}), 7.24-7.28$ (m, 1H), 7.20-7.22 (d, $J=8.0,1 \mathrm{H}), 6.92-$ $6.94(\mathrm{~m}, 1 \mathrm{H}) ;{ }^{13} \mathrm{C} \mathrm{NMR}(100 \mathrm{MHz}, \mathrm{CDCl} 3) \delta 141.1,140.0$, $139.7,139.5,137.9,137.6,135.6,133.21,133.17,130.3$, $128.9,128.2,127.8,127.6,127.4,126.9,126.5,126.3,125.8$, $125.4,125.34,125.28,125.2,124.0,123.8,123.7,122.8$, 122.1, 120.8, 120.5, 93.4, 87.6; IR (ATR) $\operatorname{vmax}\left(\mathrm{cm}^{-1}\right)$ : 636.34, 732.63, 759.06, 1435.42, 2199.58, 2923.58, 2980.52, 3055.86; HRMS calcd for $\mathrm{C}_{32} \mathrm{H}_{19} \mathrm{~S}_{3}, 499.0649[\mathrm{M}+\mathrm{H}]^{+ \text {; }}$ found $499.0575[\mathrm{M}+\mathrm{H}]^{+}$.

3-(3-(6-Methoxynaphthalen-2-yl)benzo[b]thiophen-2-yl)2-(thiophen-2-yl)benzo[b]thiophene 15D was isolated in $\mathbf{5 8 \%}$ yield as a yellow solid: ${ }^{1} \mathrm{H}$ NMR $\left(400 \mathrm{MHz}, \mathrm{CDCl}_{3}\right)$ $\delta 8.12-8.14(\mathrm{~d}, J=8.0,1 \mathrm{H}), 7.85-7.91(\mathrm{~m}, 2 \mathrm{H}), 7.69-7.71$ $(\mathrm{m}, 1 \mathrm{H}), 7.52-7.64(\mathrm{~m}, 4 \mathrm{H}), 7.46-7.49(\mathrm{~m}, 1 \mathrm{H}), 7.35-7.41$ $(\mathrm{m}, 2 \mathrm{H}), 7.20-7.27(\mathrm{~m}, 3 \mathrm{H}), 7.10-7.13(\mathrm{~m}, 1 \mathrm{H}), 7.04$ $7.05(\mathrm{~d}, J=4.0,1 \mathrm{H}), 6.94-6.97(\mathrm{~m}, 1 \mathrm{H}), 3.90(\mathrm{~s}, 3 \mathrm{H})$; 13C NMR $\left(100 \mathrm{MHz}, \mathrm{CDCl}_{3}\right) \delta 158.5,140.9,140.0,139.9$, 139.0, 138.1 137.3, 135.8, 134.3, 131.5, 129.5, 129.2, 128.6, 127.7 (include 2C), 127.4, 126.8, 125.7, 125.3, 125.14, $125.10,124.2,123.89,123.86,122.7,122.1,120.2,119.5$, 118.2, 106.0, 96.0, 82.7, 55.6; IR (ATR) $\operatorname{vmax}\left(\mathrm{cm}^{-1}\right)$ : 699.57, 729.04, 766.36, 1029.85, 1196.55, 1391.35, 1600.36, 1627.59, 1732.15, 2202.73, 2926.61, 2956.69; HRMS calcd for $\mathrm{C}_{33} \mathrm{H}_{21} \mathrm{OS}_{3}, 529.0755[\mathrm{M}+\mathrm{H}]^{+;} 529.0755[\mathrm{M}+\mathrm{H}]^{+}$. 


\subsection{Synthesis of 3-(1H-indole-2-yl)-2- (thiophen-2-yl)benzo[b]thiophene (16)}

To a solution of the 2-(2-aminobenzoethynyl)-2-(thiophen-2yl) benzo $[b]$ thiophene $(\mathbf{1 2 J})(99.3 \mathrm{mg}, 0.3 \mathrm{mmol})$ in dichloromethane $(10 \mathrm{~mL})$ was added $\mathrm{I}_{2}(226.8 \mathrm{mg}, 0.9 \mathrm{mmol})$ under argon atmosphere. The mixture was stirred at room temperature for $1 \mathrm{~h}$, followed by quenching with sat. sodiumthiosulfate $(30 \mathrm{~mL})$ and extracted with DCM $(20 \mathrm{~mL} \times 3)$. The organic layer was dried over anhydrous $\mathrm{MgSO}_{4}$ and filtered. Solvent was removed under vacuum, and the residue was purified by column chromatography over silica gel with Hexane/EtOAc (10/1) to afford 3-(1H-indole-2-yl)-2(thiophen-2-yl) benzo[b]thiophene16 as a white solid in $\mathbf{6 7 \%}$ yield. M.p.: $159.8-161.4{ }^{\circ} \mathrm{C} ;{ }^{1} \mathrm{H}$ NMR $\left(400 \mathrm{MHz}, \mathrm{CDCl}_{3}\right)$ $\delta 8.02(\mathrm{~b}, 1 \mathrm{H}), 7.84-7.82(\mathrm{~m}, 1 \mathrm{H}), 7.75-7.70(\mathrm{~m}, 1 \mathrm{H})$, 7.40-7.33 (m, 3H), 7.27-7.15 (m, 4H), 6.97-6.94 (m, 1H), 6.78-6.77 (m, 1H); ${ }^{13} \mathrm{C}$ NMR $\left(100 \mathrm{MHz}, \mathrm{CDCl}_{3}\right) \delta 140.9$, $138.2,136.5,135.8,135.4,130.4,128.9,127.6,127.5,127.4$, 125.4, 125.2, 124.5, 123.6, 122.5, 122.1, 121.0, 120.3, 111.3, 104.8; IR (ATR) $v_{\max }\left(\mathrm{cm}^{-1}\right): 3444.54,2917.97,2850.71$, 1729.02, 1419.21, 1231.16, 732.22, 699.57; HRMS calcd for $\mathrm{C}_{20} \mathrm{H}_{14} \mathrm{NS}_{2}, 332.0565[\mathrm{M}+\mathrm{H}]^{+;}$found $332.0555[\mathrm{M}+\mathrm{H}]^{+}$.

\subsection{Antimicrobial activity test}

Antimicrobial acitivities of the newly synthesized compounds were evaluated against reference strains kindly provided from Refik Saydam National Type Culture Collection (Turkey): three gram positive bacteria (Bacillus subtilis ATCC 6633, Enterococcus faecalis ATCC 29212, Staphylococcus aureus ATCC 25923), three gram negative bacteria (Escherichia coli ATCC 25922, Klebsiella pneumoniae ATCC 700603, Pseudomonas aeruginosa ATCC 27853), an yeast (Candida albicans ATCC 10231) and a fungus (Aspergillus niger ATCC 16404). Initially, agar disk diffusion method was used to screen in vitro antimicrobial activities of the synthesized compounds ${ }^{28}$ on the basis of Clinical and Laboratory Standards Institute (CLSI) recommendation. The results were recorded for each tested compound as the average diameter (in $\mathrm{mm}$ ) of inhibition zones around the discs where no microbial growth occurred. The compounds that lead to significant zone diameters $(>10 \mathrm{~mm})$ against the tested organisms were further analysed quantitatively by determining Minimal Inhibitory Concentration (MIC) values. MIC corresponds to the lowest concentration required to inhibit microbial growth. Broth microdilution method in cation-adjusted Mueller-Hinton Broth (MHB) (Oxoid) was performed in 96well round-bottom microtiter plates according to the CLSI guidelines. ${ }^{29} 100 \mu \mathrm{l}$ of MHB was dispensed to each well. Then, the compounds dissolved in DMSO in a final concentration of $1024 \mu \mathrm{g} / \mathrm{mL}$ were added to first wells, and doubling dilution series were performed to get a concentration scale from $512 \mu \mathrm{g} / \mathrm{mL}$ to $0.125 \mu \mathrm{g} / \mathrm{mL}$. The standards (amikacin for bacteria and flucanozole for fungi) were diluted in the same way. The sterility, growth and solvent controls (MHB without inoculum, bacteria in the absence of the compounds and dimethylsulfoxide (DMSO) diluted in the medium, respectively) were used, as well. Freshly prepared streak plated microorganisms were resuspended in $0.85 \% \mathrm{NaCl}$ to 0.5 McFarland $\left(\cong 1-4 \times 10^{8} \mathrm{CFU} / \mathrm{mL}\right) .100 \mu \mathrm{L}$ of this solution was tenfold diluted in MHB and next microbial inoculum was added to each well (as final concentration of $1-5 \times 10^{5} \mathrm{CFU} / \mathrm{mL}$ ). Incubation was done at $37^{\circ} \mathrm{C}$ for $16-$ $20 \mathrm{~h}$ for bacteria and $26^{\circ} \mathrm{C}$ for $24-48 \mathrm{~h}$ for fungi. All MIC determinations were carried out in triplicates. The MIC values of the tested compounds were detected visually by controlling the turbidity with respect to the controls.

\subsection{Total antioxidant capacity test by ABTS assay}

2.13a ABTS assay: ABTS, 2, 2' - azinobis (3-ethylbenzothiazoline-6-sulfonic acid) diammonium salt, potassium persulfate and ethanol were purchased from Sigma-Aldrich. All chemicals and solvents were analytical grade and were used as received. Antioxidant capacities of the synthesized chemicals were determined according to the modified method of Rea et al., ${ }^{30}$ Trolox was used as the antioxidant standard. ABTS stock solution, including ABTS $(7 \mathrm{mM})$ and potassium persulphate $(2,45 \mathrm{mM})$, was prepared in distilled $\mathrm{H}_{2} \mathrm{O}$ and incubated at room temperature for $12-16 \mathrm{~h}$. Then, ABTS stock solution was diluted with ethanol to achieve an absorbance of $0.700 \pm 0.02$ at $734 \mathrm{~nm}$ to be used as working solution. The synthesized dibenzothiophenes and trolox were dissolved and diluted with pure ethanol. After that, trolox standard or our compounds were mixed with ABTS working solution (1:1) and incubated in the dark at $25^{\circ} \mathrm{C}$ for $30 \mathrm{~min}$ with continuous moderate shaking. The reaction steps were also repeated for controls containing ethanol and ABTS. Absorbances were measured at $734 \mathrm{~nm}$. All measurements were done triplicated under dim light. This procedure was repeated three times at different dates.

The percentage of Radical Scavenging Activity (\%RSA) was calculated using the following equation:

$$
\begin{aligned}
& \text { Radical Scavenging Activity (\%RSA) } \\
& =\frac{(\text { Control Absorbance }- \text { Sample Absorbance })}{(\text { Control Absorbance })} \times 100
\end{aligned}
$$

The $\mathrm{IC}_{50}$ value is the concentration of compound that is able to reduce the absorbance value of the $\mathrm{ABTS}^{+}$radical cation solution to half of its original value. This value obtained from linear curve of RSA\% versus different concentrations of tested compound. If the $\%$ RSA value could not exceed $50 \%$ to be used in the calculation of $\mathrm{IC}_{50}$ value, then $\%$ RSA value itself was used as a unit in the evaluation of the total antioxidant capacity after calculation of the ratio with the applied concentration of the molecules (\% RSA/applied concentration, $\mathrm{mL} / \mathrm{mg}$ ). 


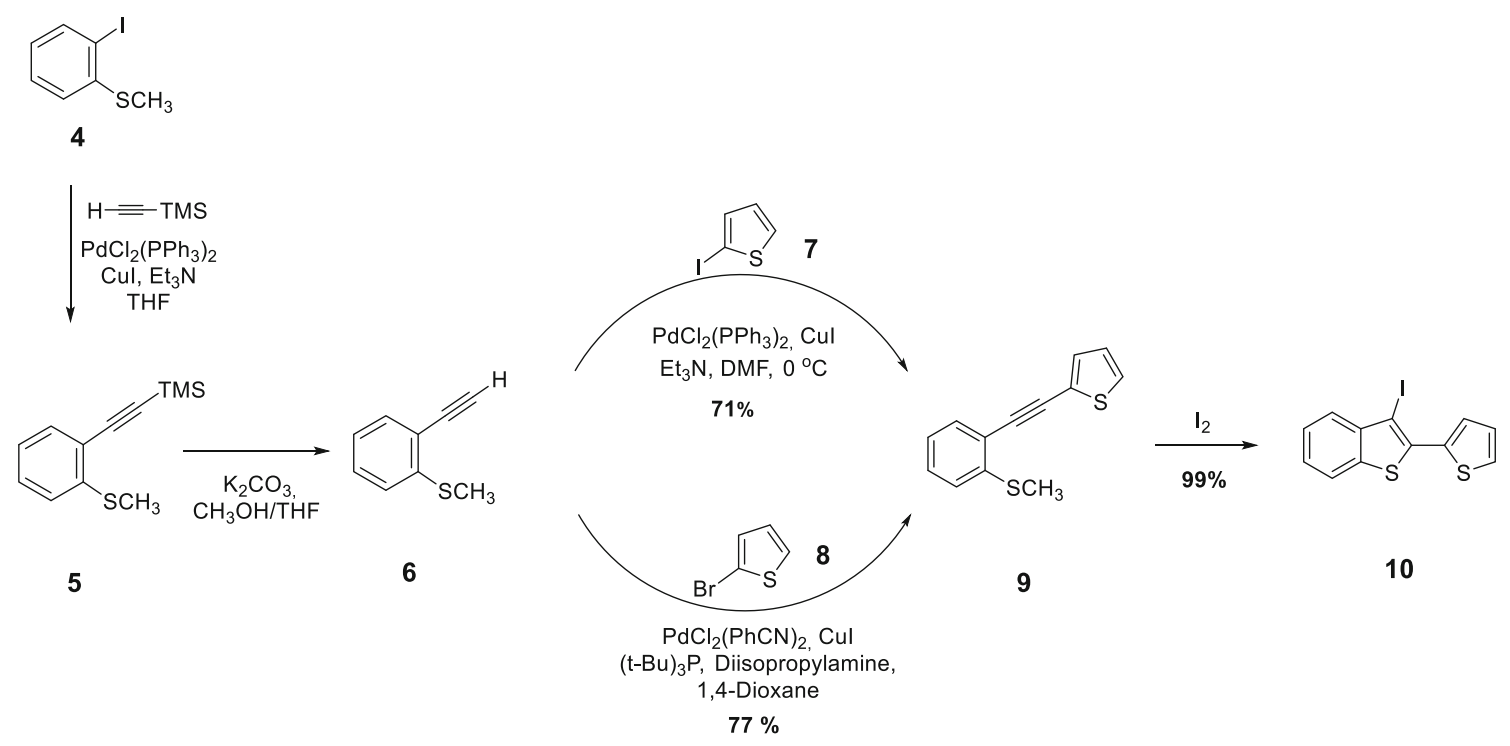

Scheme 2. Synthesis of 3-iodo-2-(thiophen-2-yl) benzo[b] thiophene (10).

\section{Results and Discussion}

\subsection{Synthesis}

Initially, palladium catalyzed Sonogashira Coupling reaction between 2-iodothioanisole $\mathbf{4}$ and trimethylsilylacetylene was performed for the preparation of 2-trimethylsilanylethynylthioanisole $\mathbf{5}$ under mild reaction conditions. After the isolation of compound 4, the trimethylsilyl group was removed by desilylation with potassium carbonate in methanol for the formation of necessary terminal alkyne as a 2-ethynylthioanisole 5. When compound 5 was allowed to react with 2bromothiophene in the presence of $\mathrm{PdCl}_{2}(\mathrm{PhCN})_{2}, \mathrm{CuI}$, $(\mathrm{t}-\mathrm{Bu})_{3} \mathrm{P}$ in Dioxane at room temperature for $12 \mathrm{~h}$, desired alkyne 9 was obtained in $77 \%$ yield. Interestingly, when 2-iodothiophene was applied for the synthesis of 9 in the presence of Pd-catalyst at room temperature, only self-coupling product of terminal alkyne was obtained instead of $\mathbf{9}$. The same reaction condition was reapplied with 2-iodothiophenes at $0{ }^{\circ} \mathrm{C}$, so that the desired compound 9 was isolated in $72 \%$ yield.

Iodine-mediated electrophilic cyclization reactions have been used for the regioselectively synthesis of five or six-membered heterocyclic compounds. ${ }^{31,32}$ Recently, Zora et al., have found a novel methodology for the synthesis of iodo-substituted-pyrazoles from corresponding acetylenic hydrazone derivatives via electrophilic cyclization under mild reaction conditions. ${ }^{33}$ Previous studies showed that $\mathrm{CH}_{2} \mathrm{Cl}_{2}$ (DCM) and chloroform were among the most employed solvents for those kinds of cyclization, and different iodine reagents such as molecular iodine, monoiodochloride
(ICl), $\mathrm{PhSeCl}$, etc. were used as electrophiles for electrophilic cyclization reactions. ${ }^{34}$ Molecular iodine is chosen being as a mild and nontoxic electrophile which catalyzed various organic reactions with high efficiency and selectivity. When 9 was reacted with molecular iodine in DCM for $2 \mathrm{~h}$ at room temperature, our expected product 3-iodo-2-(thiophen-2-yl)benzo[b]thiophene (10) was obtained in good yield (99\%) (Scheme 2).

After isolation of structure 10, a variety of 3-alkynylic benzothiophene derivatives were synthesized by using Pd-catalyzed Sonogashira Cross-Coupling reactions. ${ }^{35}$ As seen in Table 1, when $\mathbf{1 0}$ was allowed to react with phenyl acetylene in the presence of $\operatorname{Pd}\left(\mathrm{PPh}_{2}\right)_{2} \mathrm{Cl}_{2}$ and $\mathrm{CuI}$ in $\mathrm{DMF} / \mathrm{Et}_{3} \mathrm{~N}$ at room temperature for overnight, 12A was obtained in $94 \%$ yield (Table 1). When the same reaction condition was applied for the synthesis of $\mathbf{1 2 B}$, the isolated yield was found as $66 \%$. When the effect of aliphatic compound was tested by using heptanyl, the $82 \%$ yield of $\mathbf{1 2 C}$ was reached. Notably, 12D was obtained in moderate yield (71\%). Moreover, amin-substituted $\mathbf{1 2 E}$ and $\mathbf{1 2 J}$ were synthesized in high yields $92 \%$ and $91 \%$, respectively, and polyaromatic substituted-12H was prepared in $93 \%$ yield. When the same reaction was performed with ethynyltrimethylsilane, $\mathbf{1 2 K}$ was formed with $89 \%$ yield. Then, 12K underwent desilylation reaction with potassium carbonate in methanol to give the new benzothiophene substituted terminal alkyne 12L which might be used as a novel precursor for the formation of biologically active compounds. As a result of the first part of study, Sonogashira cross coupling reactions were found to be general for a wide range of terminal alkynes and tolerated the presence of aliphatic, 
Table 1. Synthesis of 3-(substituted-ethynyl)-2-(thiophen-2-yl) benzo[b]thiophene (12) derivatives.

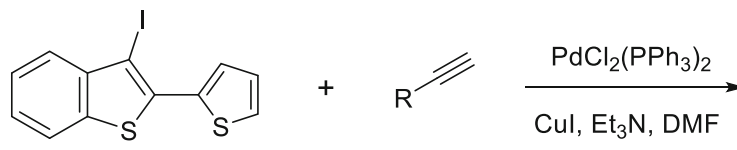

10
11

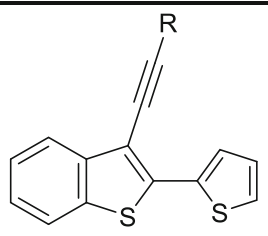

12

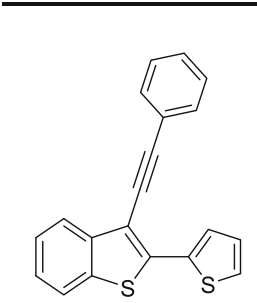

$12 \mathrm{~A}(94 \%)$<smiles>Cc1ccc(C)c(C#Cc2c(-c3cccs3)sc3ccccc23)c1</smiles>

$12 \mathrm{G}(69 \%)$

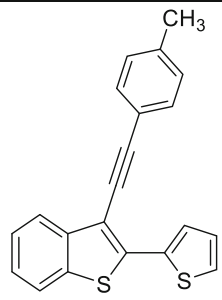

12B $(66 \%)$

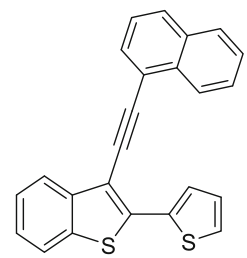

$12 \mathrm{H}(93 \%)$

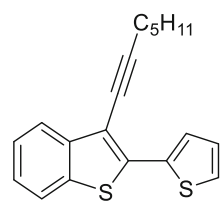

$12 \mathrm{C}(82 \%)$

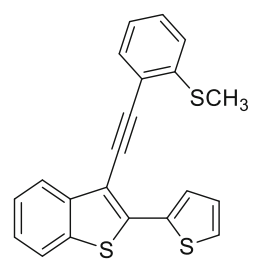

$121(81 \%)$

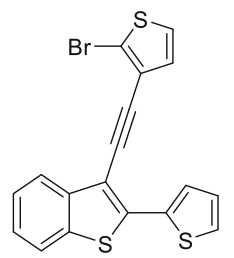

12D (71\%)
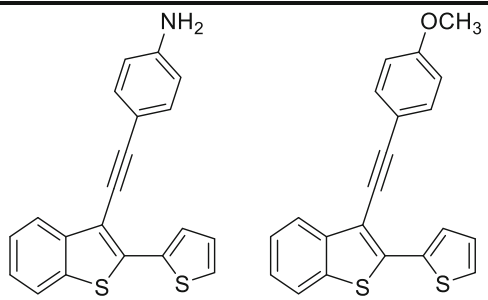

$12 \mathrm{E}(92 \%)$

$12 \mathrm{~F}(70 \%)$

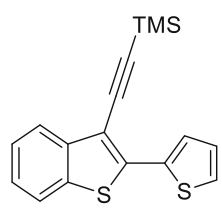

$12 \mathrm{~K}(89 \%)$

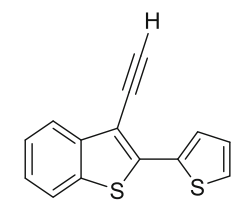

$12 L(83 \%)$

$12 \mathrm{~J}(91 \%)$

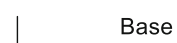

aromatic, polyaromatic and heteroaromatic moieties with electron-withdrawing and electron-donating substituents (Table 1).

After preparation of 3-(substituted-ethynyl)-2(thiophen-2-yl) benzo[b]thiophenes (12A-L), the synthesis of corresponding iodo substituted-thienodibenzothiophene (13) was examined by using iodinecatalyzed electrophilic cyclization reaction (S. I.). Initially, 12A was allowed to react with 1 equiv. of molecular iodide in DCM at room temperature for $24 \mathrm{~h}$, all starting compound was recovered. When excess amount of iodide was used in the same reaction conditions, there was no formation of products.

When 12I and 12J were allowed to cyclization reaction in the presence of molecular iodine, $\mathbf{1 4}$ and 16 were obtained in $81 \%$ and $67 \%$ yields, respectively (Schemes 3 and 4). Although, Larock et al., reported the synthesis of 3-benzofuran-substituted-2phenyl-benzothiophene via electrophilic cyclization reactions, there is not any information on indoles or benzothiophene-susbtituted benzothiophene structures

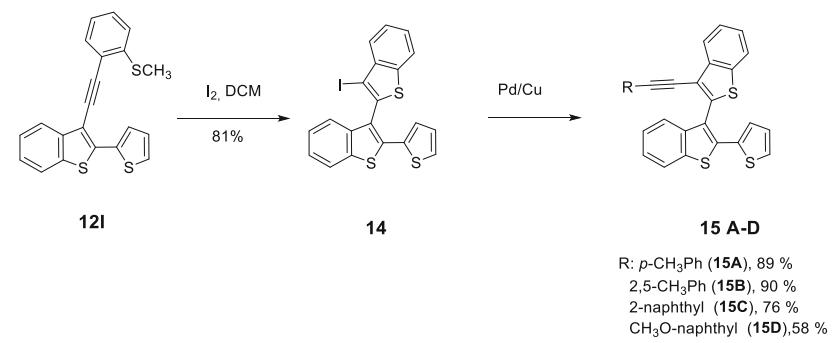

Scheme 3. Synthesis of 3-(2-thioanisolethynyl)-2-(thiophen-2-yl)benzo[b]thiophene (12I) and 3-(arylethynyl)- $2^{\prime}$ (thiophen-2-yl)-2,3'-bibenzo[b]thiophene (15 A-D).

in literature. ${ }^{36}$ The methodology proposed in current study gave the opportunity to the synthesis of novel heteroaromatic compounds including two benzothiophenes and benzothiophenes-indoles. Interestingly, the cyclization reaction of $\mathbf{1 2 J}$ produced the only indole (16) instead of iodine-substituted corresponding product (Scheme 4). Moreover, 15A-D were synthesized 


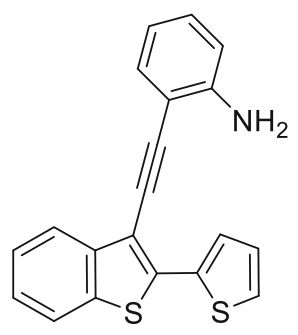

12J

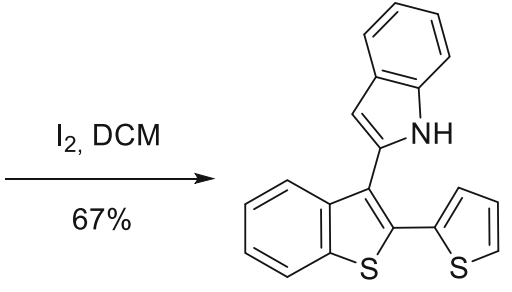

16
Scheme 4. Synthesis of 2-(2-(thiophen-2-yl) benzo[b] thiophen-3-yl)-1H-indole.

from 14 by using palladium catalyzed Sonogashira cross coupling reactions (Scheme 4).

\subsection{Evaluation of antimicrobial activities of the newly synthesized compounds}

In the present study, antimicrobial activities of new benzothiophene derivatives were tested against indicator microorganisms by using agar disc diffusion method (Table S2, SI). When the zone inhibition values were compared to interpretive standards (CLSI), the compounds (9) (againstC. albicans ATCC 10231), (10) (against B. subtilis ATCC 6633, E. coli ATCC 25922, K. pneumoniae ATCC 700603, C. albicans ATCC 10231 and $A$. niger ATCC 16404), (6), (12E), (12J) and (12L) (against $S$. aureus ATCC 25923), and (12K) (against A. niger ATCC 16404) were found to have considerable antimicrobial activities and their MIC values were determined by microdilution method (Table S3, Supplementary Information). According to the inhibition zone and MIC data, 2-trimethylsilanylethynylthioanisole (5) was not found to be effective against tested microorganisms. On the other hand, structure $\mathbf{6}$ gave slightly higher inhibition zone value corresponding to a weak antibacterial activity (MIC of $256 \mu \mathrm{g} / \mathrm{mL}$ ) against $S$. aureus ATCC 25923, a gram positive bacterium. Compound 9 displayed a weak antifungal activity only for C. albicans ATCC 10231. Compound 10, obtained from electrophilic cyclization, showed good antibacterial and antifungal activities against B. subtilis ATCC 6633 and A. niger ATCC 16404 with MIC values of 42,7 and $128 \mu \mathrm{g} / \mathrm{ml}$, respectively; and, expanded its activity spectrum mildly against $E$. coli ATCC $25922, K$. pneumoniae ATCC 700603 and C. albicans ATCC 10231 (MIC values of $250 \mu \mathrm{g} / \mathrm{mL}$ ). The isolated molecules (12A-L) exhibited the highest antimicrobial activity against $S$. aureus ATCC 25923 and A. niger ATCC 16404. 12E, 12J and 12L were found to be the most potent antibacterial compound against $S$. aureus ATCC 25923 with MIC values of $4-5,3,16$ and $4 \mu \mathrm{g} / \mathrm{mL}$, respectively. The highest antifungal activity against $A$. niger ATCC 16404 (MIC: $64 \mu \mathrm{g} / \mathrm{ml}$ ) was observed for compound $12 \mathrm{~K}$. As a summary, our novel benzothiophenes seem to be significantly effective on gram positive bacteria, $S$. aureus ATCC 25923 and B. subtilis ATCC 6633; and fungi, C. albicans ATCC 10231 and A. niger ATCC 16404. The compounds 12E, 12L and 12J with potent antibacterial activities against $S$. aureus might be new candidates for the development of a new class of antimicrobial agents in treatment of multidrug resistant strains. Similarly, compound $\mathbf{1 0}$ and $\mathbf{1 2 K}$ might be further modified to design potent antifungal agents against current fungal diseases.

\subsection{Assessment of antioxidant capacities of the newly synthesized compounds}

Trolox is a water soluble vitamin $\mathrm{E}$ analogue $\mathrm{e}^{37}$ and those analogues are used in food industry and medicine because of being good chain-breaking antioxidants which prevent peroxidation of lipids and also being adventageous stabilizers of biological membranes via the restriction of components' mobilities in membranes. In this manner, a new compound with higher antioxidant capacity than that of trolox is a good candidate for the development of better ingredients in those industrial areas.

Antioxidant capacities of newly synthesized dibenzothiophenes were tested by a universal consent method of Trolox Equivalent Antioxidant Capacity Assay (TEAC) as defined before and the results indicated that among all derivatives, amine-substituted derivative of 3-(substituted-ethynyl)-2-(thiophen-2-yl) benzo[b] thiophene $(\mathbf{1 2 E})$ and the product of the cyclization of a homologues of this analog in the presence of iodine (16) had the highest antioxidant capacities as compared to trolox (Table S4, Supplementary Information).

Almost one of third of the newly synthesized dibenzothiophene derivatives exerted strong radical scavenging activities in vitro (TEAC assay) and $\mathrm{IC}_{50}$ values could be calculated. Compound $\mathbf{1 6}$ was determined to be 2.5 times more potent than trolox (Table S5, Supplementary Information) and it made this derivative more powerful not only against vitamin E derivatives like trolox but also against many other phenolic compounds such as ascorbic acid, chlorogenic acid, phloridzin, ${ }^{38}$ and caffeic acid ${ }^{39}$ found in food supplies and medicines. Additionaly, the derivative 12E, also, exhibited a slightly higher TEAC value than trolox which made it a significant antioxidant, too.

In summary, except the highly outstanding derivatives $\mathbf{1 6}$ and $\mathbf{1 2 E}$, the others that could be used in the 
development of better antioxidant compounds, but still having lower TEAC values than trolox standard (Table S5, Supplementary Information), showed a sorting as: 12J $>12 \mathrm{D}>9>6$.

\section{Conclusions}

In this study, novel benzothiophene derivatives were designed, synthesized and investigated for some of their biological properties. Our compounds have special structures of molecules that possessed interesting biological properties and pharmaceutical potentials. It was found that 2-(thiophen-2-yl) benzo[b]thiophene (12E), 3-ethynyl-2-(thiophen-2-yl) benzo[b]thiophene (12L) and 3-(2-aminobenzoethynyl)-2-(thiophen-2-yl) benzo[b]thiophene (12J) have high antibacterial activities against $S$. aureus ATCC 25923 which is a very dangerous temporizer pathogenic bacterium colonized on one of every five human living on the world. Moreover, 3-iodo-2-(thiophen-2-yl) benzo[b]thiophene (10) and 3(trimethylsilylethynyl)-2-(thiophen-2-yl) benzo[ $b]$ thiophene $(\mathbf{1 2 K})$ could be used for the treatment of fungal diseases caused by A. niger ATCC 16404 . The derivatives 16 and $\mathbf{1 2 E}$ displayed quite high antioxidant capacities with TEAC values of 2.5 and 1.1, respectively. Finally, the synthesized novel compounds possessed interesting positions which could work as keys for further verifying reactions to increase the number of new derivatives in future studies.

\section{Supplementary Information (SI)}

Characterization of the new benzo[b]thiophene products including Scheme 2, Table S2, S3, S4 and S5, ${ }^{1} \mathrm{H}$ NMR, ${ }^{13} \mathrm{C}$ NMR, and FTIR spectra, as well as mass characterization data. Supplementary Information is available on www.ias.ac. in/chemsci.

\section{Acknowledgements}

The authors thank to The Scientific and Technological Research Council of Turkey (Project No: 115Z020) for financial supporting of silica gels, column chromatographic solvents, all other reactants and reagents. Authors, also, thank Van Yüzüncü Yil University (Project No: FBA-2017-6007) for financial supporting of glassware and salts and University of Duhok for a scholarship to Muheb A. S. Algso.

\section{References}

1. Brasholz M, Reissig H U and Zimmer R 2009 Sugars, alkaloids, and heteroaromatics: Exploring heterocyclic chemistry with alkoxyallenes Acc. Chem. Res. 4245
2. Kivrak A and Larock R C 2010 Synthesis of dihydrobenzisoxazoles by the $3+2$ cycloaddition of arynes and oxaziridines J. Org. Chem. 757381

3. Coa J C, Castrillon W, Cardona W, Carda M, Ospina V, Munoz J A, Velez I D and Robledo S M 2015 Synthesis, leishmanicidal, trypanocidal and cytotoxic activity of quinoline-hydrazone hybrids Eur. J. Med. Chem. 101 746

4. Shakdofa M M E, Shtaiwi M H, Morsy N and AbdelRassel T M A 2014 Metal complexes of hydrazones and their biological analytical and catalytic applications: A review Main Group Chem. 13187

5. Rahmouni A, Souiei S, Belkacem M A, Romdhane A, Bouajila J and Ben Jannet H 2016 Synthesis and biological evaluation of novel pyrazolopyrimidines derivatives as anticancer and anti-5-lipoxygenase agents Bioorg. Chem. 66160

6. Pathak R B, Chovatia P T and Parekh H H 2012 Synthesis, antitubercular and antimicrobial evaluation of 3-(4chlorophenyl)-4-substituted pyrazole derivatives Bioorg. Med. Chem. Lett. 225129

7. Kazemizadeh A R, Shajari N, Shapouri R, Adibpour N and Teimuri-Mofrad R 2016 Synthesis and anti-brucella activity of some new 1,3,4-oxadiazole derivatives containing a ferrocene unit J. Iran. Chem. Soc. 131349

8. Richardson D R, Kalinowski D S, Lau S, Jansson P J and Lovejoy D B 2009 Cancer cell iron metabolism and the development of potent iron chelators as anti-tumour agents Biochim. Biophys. Acta 1790702

9. Williams R J, Spencer J P E and Rice-Evans C 2004 Flavonoids: Antioxidants or signalling molecules? Free Rad. Biol. Med. 36838

10. Edwards G and Weston A H 1993 The pharmacology of ATP-sensitive potassium channels Annu. Rev. Pharmacol. Toxicol. 33597

11. Pommier Y 2016 Topoisomerase I inhibitors: Camptothecins and beyond Nat. Rev. Cancer 6789

12. Grynyuk, II, Prylutska S V, Franskevych D V, Trush V A, Sliva T Y, Slobodyanik M S, Hurmach V V, Prylutskyy Y I, Matyshevska O P and Ritter U 2016 Combined action of $\mathrm{C}_{60}$ fullerene with dimethyl-N-(benzoyl)amidophosphate or dimethyl-N(phenylsulfonyl) amidophosphate on leukemia L1210 cells in silico and in vitro Materialwissenschaft und Werkstofftechnik $\mathbf{4 7} 98$

13. Meixner C N, Aref M W, Gupta A, McNerny E M B, Brown D, Wallace J M and Allen M R 2017 Raloxifene Improves Bone Mechanical Properties in Mice Previously Treated with Zoledronate Calcif. Tissue Int. 101 75

14. Sarret C, Pichard S, Afenjar A and Boespflug-Tanguy O 2017 Lack of long-term neurologic efficacy of zileuton in Sjogren-Larsson's syndrome Neuropediatrics $\mathbf{4 8} 205$

15. Croxtall J D and Plosker G L 2009 Sertaconazole: A review of its use in the management of superficial mycoses in dermatology and gynaecology Drugs 69339

16. Vogel V G, Costantino J P, Wickerham D L, Cronin W M, Cecchini R S, Atkins J N, Bevers T B, Fehrenbacher L, Pajon E R, Wade J L, Robidoux A, Margolese R G, James J, Lippman S M, Runowicz C D, Ganz P A, Reis S E, McCaskill-Stevens W, Ford L G, Jordan V C, Wolmark $\mathrm{N}$ and NSABP 2006 Effects of tamoxifen vs raloxifene 
on the risk of developing invasive breast cancer and other disease outcomes: The NSABP study of tamoxifen and raloxifene (STAR) P-2 trial JAMA 2952727

17. Liu S, Zha C, Nacro K, Hu M, Cui W, Yang Y L, Bhatt U, Sambandam A, Isherwood M, Yet L, Herr M T, Ebeltoft S, Hassler C, Fleming L, Pechulis A D, Payen-Fornicola A, Holman N, Milanowski D, Cotterill I, Mozhaev V, Khmelnitsky Y, Guzzo P R, Sargent B J, Molino BF, Olson R, King D, Lelas S, Li Y-W, Johnson K, Molski T, One A, Ng A, Haskell R, Clarke W, Bertekap R, O'Connell J, Lodge N, Sinz M, Adams S, Zaczek R and Macor J E 2014 Design and synthesis of 4-heteroaryl 1,2,3,4-tetrahydroisoquinolines as triple reuptake inhibitors ACS Med. Chem. Lett. 5760

18. Sun L L, Deng C L, Tang R Y and Zhang X G 2011 CuI/TMEDA-catalyzed annulation of 2-bromo alkynylbenzenes with $\mathrm{Na}_{2} \mathrm{~S}$ : Synthesis of benzo b thiophenes $J$. Org. Chem. 767546

19. Rafiq S M, Sivasakthikumaran R and Mohanakrishnan A K 2014 Lewis acid/bronsted acid mediated benzannulation of thiophenes and electron-rich arenes Org. Lett. 162720

20. Bartlett J G, Gilbert D N and Spellberg B 2013 Seven ways to preserve the miracle of antibiotics Clin. Infect. Dis. 561445

21. Morry J, Ngamcherdtrakul W and Yantasee W 2017 Oxidative stress in cancer and fibrosis: Opportunity for therapeutic intervention with antioxidant compounds, enzymes, and nanoparticles Redox Biol. 11240

22. Marrazzo G, Barbagallo I, Galvano F, Malaguarnera M, Gazzolo D, Frigiola A, D'Orazio N and Volti G L 2014 Role of dietary and endogenous antioxidants in diabetes Crit. Rev. Food Sci. Nutr. 541599

23. Myung S K, Ju W, Cho B, Oh S W, Park S M, Koo B K and Park B J 2013 Efficacy of vitamin and antioxidant supplements in prevention of cardiovascular disease: Systematic review and meta-analysis of randomised controlled trials BMJ 346

24. Sesso H D, Buring J E, Christen W G, Kurth T, Belanger C, MacFadyen J, Bubes V, Manson J E, Glynn R J and Gaziano J M 2008 Vitamins E and C in the prevention of cardiovascular disease in men the physicians' health study II randomized controlled trial JAMA 3002123

25. Wojcik M, Burzynska-Pedziwiatr I and Wozniak L A 2010 A review of natural and synthetic antioxidants important for health and longevity Curr. Med. Chem. 17 3262

26. Chen C C, Chen C M and Wu M J 2014 Transition metal-catalyzed cascade cyclization of aryldiynes to halogenated benzo b naphtho 2,1-d thiophene derivatives J. Org. Chem. 794704
27. Lu W D and Wu M J 2007 Halocyclization of 2-alkynylthioanisoles by cupric halides: Synthesis of 2substituted 3-halobenzo b thiophenes Tetrahedron 63 356

28. Desai R M, Dodiya D K, Trivedi A R and Shah V H 2008 Simple and efficient synthetic routes to bioactive striazinyl dithiocarbamate derivatives Med. Chem. Res. 17 495

29. Gu W and Wang S F 2010 Synthesis and antimicrobial activities of novel $1 \mathrm{H}$-dibenzo a,c carbazoles from dehydroabietic acid Eur. J. Med. Chem. 454692

30. Re R, Pellegrini N, Proteggente A, Pannala A, Yang M and Rice-Evans C 1999 Antioxidant activity applying an improved ABTS radical cation decolorization assay Free Rad. Bio. Med. 261231

31. Kumar S, Mujahid M and Verma A K 2017 Regioselective 6-endo-dig iodocyclization: An accessible approach for iodo-benzo a phenazines Org. Biomol. Chem. 15 4686

32. Miao M Z, Xu H P, Luo Y, Jin M C, Chen Z K, Xu J F and Ren H J 2017 A modular approach to highly functionalized 3-sulfonylfurans via conjugate addition of 3-cyclopropylideneprop-2-en-1-ones with sodium sulfinates and sequential 5-endo-trig iodocyclization Org. Chem. Frontiers 41824

33. Zora M, Kivrak A and Yazici C 2011 Synthesis of pyrazoles via electrophilic cyclization J. Org. Chem. 76 6726

34. Togo H and Iida S 2006 Synthetic use of molecular iodine for organic synthesis Synlett 2159

35. Chinchilla R and Najera C 2007 The sonogashira reaction: A booming methodology in synthetic organic chemistry Chem. Rev. 107874

36. Mehta S and Larock R C 2010 Iodine/palladium approaches to the synthesis of polyheterocyclic compounds J. Org. Chem. 751652

37. Lucio M, Nunes C, Gaspar D, Ferreira H, Lima J and Reis S 2009 Antioxidant activity of vitamin E and trolox: Understanding of the factors that govern lipid peroxidation studies in vitro Food Biophys. 4312

38. Miller N J and Rice E C A 1997 The relative contributions of ascorbic acid and phenolic antioxidants to the total antioxidant activity of orange and apple fruit juices and blackcurrant drink Food Chem. 60331

39. Ozgen M, Reese R N, Tulio A Z, Scheerens J C and Miller A R 2006 Modified 2,2-azino-bis-3ethylbenzothiazoline-6-sulfonic acid (ABTS) method to measure antioxidant capacity of selected small fruits and comparison to ferric reducing antioxidant power (FRAP) and 2,2'-diphenyl-1-picrylhydrazyl (DPPH) methods $J$. Agricul. Food Chem. 541151 